\title{
ANTIRETROVIRAL TREATMENT IN RESOURCE-POOR SETTINGS: A VIEW FROM INDIA
}

\author{
MADHU VAJPAYEE, SHWETA KAUSHIK, KAMALIKA MOJUMDAR, V. SREENIVAS*
}

\section{ABSTRACT}

CONTEXT: The introduction of highly effective generic antiretroviral drugs at reduced cost has transformed the face of HIV/AIDS epidemic in developing countries like India. However, there is an urgent emphasis on developing and implementing guidelines for antiretroviral treatment monitoring by laboratory methods utilizing the available technologies in resource-limited settings. AIM: We studied the efficacy of antiretroviral treatment, adherence to therapy and motivation of patients for regular treatment monitoring by CD4 counts. SETINNGS AND DESIGN: A longitudinal cohort study on an established cohort of 166 HIV-1-infected Indian individuals. MATERIALS AND METHODS: Study subjects were followed up for the period from January 2002 to November 2006. Their clinical status and treatment regimen were recorded, and CD4 counts were performed at each visit. STATISTICAL ANALYSIS: Repeated-measures ANOVA was used to compute changes in median CD4 counts at each visit in the different treatment groups. RESULTS: We observed a growing awareness and motivation for regular HIV disease monitoring among patients, accompanied by a trend of increasing median CD4 counts at all subsequent follow-up visits after initiation of antiretroviral treatment. CONCLUSIONS: The study gives an insight into the institutional efforts for the establishment of cohorts for longitudinal studies, which will help in designing effective treatment guidelines, thus providing impetus to the free public sector antiretroviral therapy program in India. Such formative research aims to fill the lacunae in the limited available data for the formulation of treatment-monitoring guidelines in resource-poor settings of developing countries like India.

Key words: Antiretroviral therapy, human immunodeficiency virus-1, India, resourcepoor

The devastating social and economic impact of ! human immunodeficiency virus (HIV)/acquired ! immunodeficiency syndrome (AIDS) pandemic !

Departments of Microbiology and *Biostatistics, All ! India Institute of Medical Sciences (A.I.I.M.S.), New ! Delhi, India!

\section{Correspondence:}

Dr. Madhu Vajpayee, !

HIV and Immunology Division, Department of Microbiology,!

All India Institute of Medical Sciences (A.I.I.M.S.), !

Ansari Nagar, New Delhi - 110 029, India.

E-mail: mvajpayee@ hotmail.com! has led to the launch of HIV-prevention! campaigns emphasizing the administration ! of powerful antiretroviral drugs. However, the ! guidelines to monitor antiretroviral therapy in ! the developed world rely heavily on laboratory ! tests based on advanced technologies that may! not be feasible or available in resource-poor ! settings. ${ }^{[1]}$ Hence it is imperative to identify and ! design monitoring strategies using the available ! technologies in resource-limited settings with a ! 
conceptual framework that takes into account the differences between rich and poor countries and provide the greatest benefit to HIV-infected! people in the developing world. ${ }^{[2-8] !}$

\section{MATERIALS AND METHODS}

The present study was a longitudinal cohort study conducted at the AIDS clinic of a tertiary ! care hospital. The study population of 166 HIV-infected individuals was referred to the AIDS clinic from the outpatient clinics of different departments in the hospital. All the 166 study subjects had at least two follow-ups ! after initial visit, at approximately 6-8 months' interval during the period January 2002 to November 2006. The appropriate institutional ethics committee approved the study, and all ! the patients gave written and informed consent ! prior to entering the study. !

The HIV antibody status of all the study subjects was assessed by three ERS [enzyme-! linked immunosorbent assay (ELISA), rapid, simple] as recommended by the National AIDS Control Organization (NACO), Ministry of Health and Family Welfare, Government of India. ${ }^{\left[{ }^{9]}\right.}$ All the tests were performed according ! to the manufacturer's recommendations. First and second-line antiretroviral-therapy regimen consisted of highly active antiretroviral therapy (HAART) or dual therapy of nucleoside/! nucleotide reverse transcriptase inhibitors (NRTIs) and non-nucleoside reverse transcriptase inhibitors (NNRTIs) or protease inhibitors (PIs), following WHO guidelines, as recommended by the Indian government. The diagnosis of pulmonary TB was based on consistent history and physical examination, positive sputum test for acid-fast bacilli and! radiologic features compatible with $\mathrm{TB} \cdot{ }^{[10]}$ ! Extrapulmonary tuberculosis, but not pulmonary ! tuberculosis, was considered an AIDS-defining ! illness. ${ }^{[11]}$ The tuberculosis regimen consisted ! of 2 months of isoniazid, rifampicin ethambutol! and pyrazinamide daily, followed by 4 months ! of isoniazid and rifampin daily. ${ }^{[12] !}$

$$
\text { co }
$$

The CD4+ T-cell percentages and counts of all ! 166 HIV-infected individuals were enumerated! at each visit of the patient by dual-color ! immunophenotyping using standard whole-! blood methodology, four-tube panel: CD45 ! /CD14; CD3 /CD4; CD3 /CD8 and isotype ! controls $\lg \gamma 1 / \lg \gamma 1 .^{[13,14]}$ The specimens were! acquired and analyzed by the CELLQuest ! software on identically configured FACS Calibur! flow cytometer (Becton Dickinson, San Jose, ! USA).

! 0

The mean and median of all study variables ! were computed for all the patients at each! visit. The changes in the median CD4 counts ! at follow-up visits were observed in different ! treatment groups using a repeated-measures ! ANOVA technique. SPSS version 11.5 and! STATA 8.0 software were used for all statistical ! analysis.

\section{RESULTS}

\section{Patients' profile}

Of the 166 HIV-infected patients comprising the ! study population, 95 (57\%) had not previously ! received antiretroviral therapy, and therapy was ! initiated in these patients at their subsequent ! follow-up visits; 37 (22\%) had early-stage ! HIV disease and did not require antiretroviral ! therapy; 34 (20\%) had been receiving ! antiretroviral therapy before enrollment in the !

study. The baseline characteristics of the study ! population have been summarized in Table 1. All the 166 patients had at least three visits to the laboratory, with the initial being the baseline ! visit and subsequent two follow-up visits by November 2006. Table 2 details the distribution! of the entire study population according to the ! duration of their follow-up visits. The most ! frequent mode of transmission was found to be ! heterosexual contact - 67 (40.3\%) among the ! study participants [Figure 1].

Out of 132 study subjects that were initiated

Table 1: Baseline characteristics of human immunodeficiency virus-infected study population

\begin{tabular}{lc}
\hline Characteristics & Value \\
\hline Total no. of study subjects ! & $166 !$
\end{tabular}

Males !

Meniales ! (otal study subjects)!

Median age (Males) !

Median age (Females) !

Distribution of study population in subgroups

Patients not receiving ART !

Patients receiving ART during follow-up visits !

Patients already receiving ART on enrollment !

Median CD4 counts of study population

Total study subjects !

Patients not recelving ART

ng follow-up visits !

ART - Antiretroviral therapy!

Table 2: Distribution of study subjects $(n=166)$ according to follow-up duration

\begin{tabular}{llllll}
\hline Groups $\left(n^{*}\right)$ & Follow-up duration (months) \\
12 & 18 & 24 & 30 & 36 & Total
\end{tabular} \begin{tabular}{rrrrrr}
12 & 18 & 24 & 30 & 36 & Total \\
\hline Patients receiving ART (Time period from enrollment before !
\end{tabular} initiation of ART) !

$\begin{array}{lcccccc}\text { After 1st visit } ! & 20 & 10 & 7 & 3 & 2 & 42 ! \\ \text { After 6-8 months! } & 6 ! & 9 ! & 14 ! & 6 ! & 5 ! & 40 !\end{array}$

\section{After 6-8 mont}

After 12-16 months

follow-up!

follow-up!

Already on ART at

enrollment!

ts not receiving

ART!

Total !

$\mathrm{n}^{*}:$ Numb
therapy! treatment during follow-up visits or did not ! require treatment, 111 patients were only HIV ! infected (HIV+TB-), and 31 patients were dually ! infected with HIV and TB (HIV+TB+). The ! distribution of the study population in different! disease and treatment groups has been! detailed in Table 3.

\section{CD4 ${ }^{+}$T-cell profile}

The 109 males (66\%) in the study group of 166 ! HIV-seropositive individuals had the baseline ! mean CD4 count of 271 cells/ $\mu$ l (range: 16 to ! 1,244 ) as compared to 308 cells/ $\mu$ l (range: 28 ! to 880 ) among 57 (34\%) females. The baseline ! mean CD4 counts of the study population in ! different subgroups are presented in Table 1.

Table 4 shows the median CD4 counts of 95 ! study subjects followed up for different time ! intervals even before the initiation of therapy.

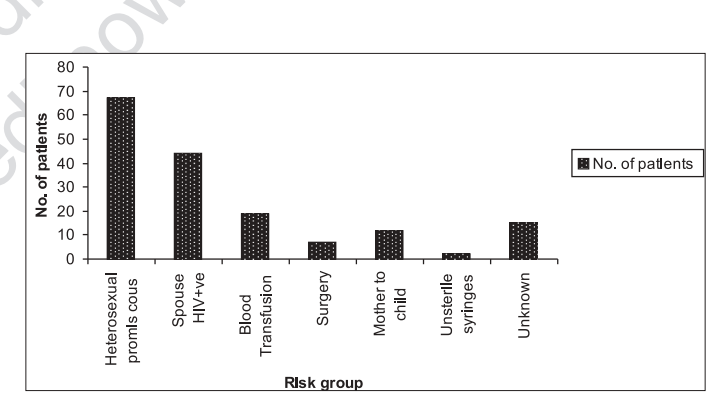

Figure 1: Distribution of study population according to ! mode of transmission!

Table 3: Distribution of study subjects $(n=132)$ in different treatment groups

\begin{tabular}{lc}
\hline Treatment group & No. of patients (\% frequency) \\
\hline Patients on ART & \\
TB+, ART alone ! & $5(3.79)$ \\
TB+, ATT alone ! & $5(3.79)$ \\
TB+, ART+ATT ! & $10(7.58)$ \\
TB-, ART+ ! & $80(60.6)$ \\
Patients not receiving ART & $1(0.76)$ \\
TB+, No Treatment ! & $31(23.48)$ \\
TB -, No Treatment ! & $132(100) !$ \\
Total &
\end{tabular}

ART - Antiretroviral therapy! 
The median CD4 counts at the last visit before! infected individuals who received treatment ! the start of antiretroviral therapy, considered as ! the baseline median CD4 counts, were $<200$ cells/ $\mu \mathrm{l}$ in all the groups of patients according to their follow-up time intervals. The median CD4 counts were also calculated at different ! follow-up time intervals for 37 HIV-infected individuals who did not require antiretroviral therapy [Table 5]. These patients maintained CD4 counts above 200 cells $/ \mu$ l at all time intervals for which they were followed up, thus ! requiring no treatment.

Changes in CD4+ T-cell counts: We observed ! an increased median CD4 T-cell count (256 cells/ $\mu$ l, range: 177 to 374 ) at the first !visit ! after initiation of antiretroviral treatment as compared to the median baseline value (179 cells/ $\mu$ l, range: 126 to 266) in 95 HIV-!

Table 4: Median CD4 counts of patients $(n=95)$ at different time intervals before the initiation Time period from Median CD4 counts (cells/ $/$ l) $[95 \%$ C.I.]

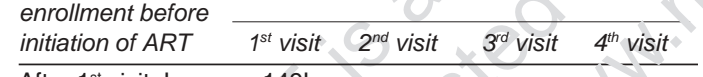
After $1^{\text {st }}$ visit $(n=42) !$
After 6-8 $(70-250) !$ months $(n=40) ! \quad \begin{array}{cc}219 ! \\ (116-298)\end{array} \quad(152-287)$

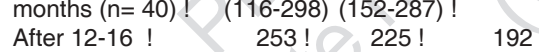
$\begin{array}{lccc}\text { After } 12-16 ! & 253 ! & 225 ! & 192 ! \\ \text { months }(\mathrm{n}=12) ! & (147-352) & (177-306) & (155-349) ! \\ \text { After 18-24! } & 329 ! & 245 ! & 182 !\end{array}$ months $(n=1)$ ! ART - Antiretroviral therapy !

AR

Table 5: Median CD4 counts at follow-up time intervals for study subjects not receiving antiretroviral therapy

\section{Time intervals of}

follow-up visits

First visit $(\mathrm{n}=37) !$

First visit $(n=37) !$

$12-16$ months $(n=37)$ !

18-24 months $(\mathrm{n}=22)$

24-32 months $(n=15)$

30-40 months ( $n=11)$

$36-48$ months $(n=5)$ !

Median CD4 counts

cells/ul) [95\% C.I.]

$364(307-528.5) !$

$394(256.5-518.5) !$

$322(249.5-504.5) !$

$368(311.3-492.8) !$

$300(216-417) !$ $336(248.5-669.5)$ ! infected individuals who received treatment !
at the follow-up visits [Table 6]. This trend of ! increasing median CD4 counts was observed! at all subsequent follow-up visits after initiation! of antiretroviral treatment as compared to ! the median baseline value. However, the ! increase in median CD4 counts after the! initiation of therapy was more apparent in ! the ffirst visit (change 1) as compared to the ! increase observed at subsequent follow-up ! visits [Figure 2]. The first increase (change ! 1) in the median CD4 counts was calculated! by subtracting the baseline CD4 count value ! from the CD4 count value at the first visit after ! initiation of antiretroviral treatment. Similarly, the ! second increase (change 2) was obtained by ! subtracting the CD4 count value at the first visit ! after initiation of antiretroviral treatment from the ! median CD4 count value at the second follow-!

Table:

Table 6: Median CD4 counts of study subjects at follow-up visits after initiation of antiretroviral therapy

\begin{tabular}{lc}
\hline $\begin{array}{l}\text { Time intervals of } \\
\text { follow-up visits }\end{array}$ & $\begin{array}{c}\text { Median CD4 counts } \\
\text { (cells/ } / \mu \text { l) }[95 \% \text { C.l. }]\end{array}$ \\
\hline $\begin{array}{l}\text { Baseline before ! } \\
\text { initiation of ART }(n=95) !\end{array}$ & $179(126-266) !$ \\
$1^{\text {ts }}$ visit $(n=95) !$ & $256(177-374) !$ \\
$2^{\text {nd }}$ visit $(n=82) !$ & $334(233.8-470) !$ \\
$3^{\text {rd }}$ visit $(n=50) !$ & $403(252.5-584.5) !$ \\
$4^{\text {th }}$ visit $(n=25) !$ & $402(267.5-664.5) !$ \\
\hline
\end{tabular}

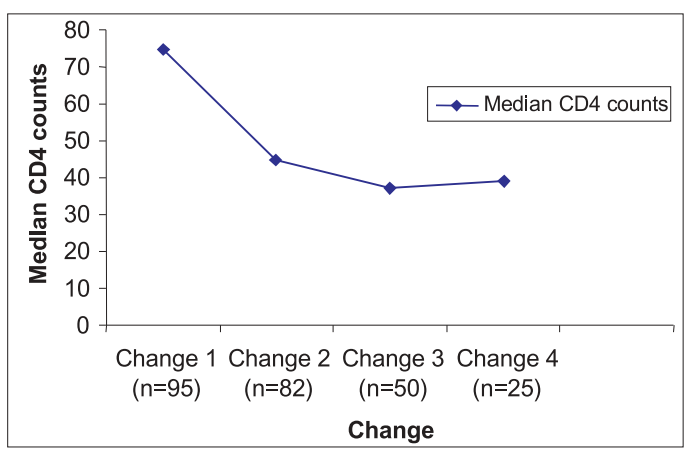

Figure 2: Change in median CD4 counts after ! initiation of antiretroviral treatment up visit after therapy; and similarly, calculations ! were done for changes at subsequent visits. ! The results also indicate that the median CD4 counts increase sharply within the first follow-! up visit after the administration of antiretroviral ! therapy and subsequently this increase is not ! quantitatively significant [Figure 2].

Effect of treatment on CD4 counts: The repeated-measures analysis of variance was! performed for 132 study subjects that were ! initiated treatment during follow-up visits or ! did not require treatment and had at least 2 follow-up visits [Figure 3]. The results indicated! trends for the increase in median CD4+ T-! cell counts at subsequent follow-up visits in ! both the groups of HIV+TB+ of 5 individuals ! and HIV+TB- of 80 individuals receiving ! only antiretroviral treatment. The group of ! $10 \mathrm{HIV}+\mathrm{TB}+$ patients receiving dual therapy ! (ART+ATT) Anti Tuberculosis Therapy (ATT) ! also showed marked improvement in median ! CD4 counts at follow-up visits. The HIV+TB+ ! group of 5 individuals receiving treatment only! for tuberculosis (ATT) had slight improvement! in CD4 counts. The HIV-seropositive individuals ! with no tuberculosis infection maintained CD4 !

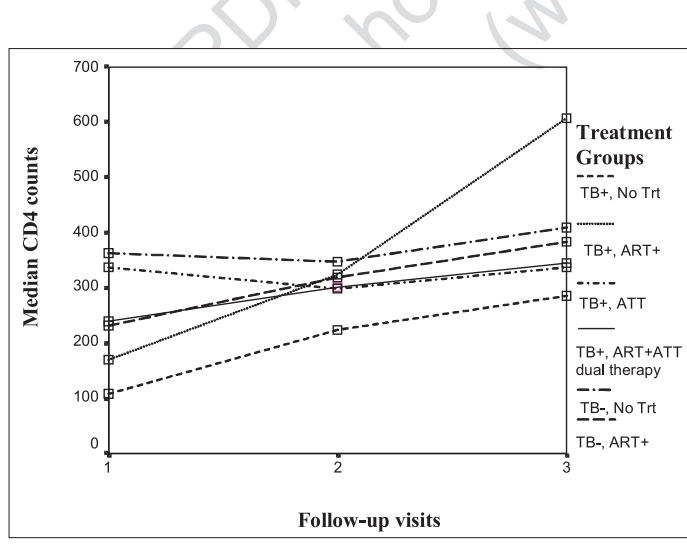

Figure 3: Changes in CD4 counts according to ! different treatment modalities! counts in absence of any treatment.

\section{DISCUSSION}

The introduction of highly effective antiretroviral drugs at affordable costs has greatly transformed the AIDS epidemic in the resource-! poor developing world. However, the effective ! delivery of antiretroviral therapy to combat! this AIDS epidemic by implementation of free ! public sector ART program requires formulation ! of guidelines for the appropriate monitoring ! of treatment effectiveness and adherence to therapy using low-cost and simple clinical ! and laboratory measures that are available in the resource-poor settings. ${ }^{[15-18]}$ The limited ! data available to develop such guidelines in ! resource-poor settings led us to analyze the efficacy of antiretroviral treatment, adherence ! to therapy and motivation of patients for ! regular treatment monitoring by CD4 counts in the already established cohort of 166 HIV-! 1 -infected Indian individuals at the All India ! Institute of Medical Sciences, New Delhi.

A significant number of individuals [95 (57\%)] within the study population had not previously ! received antiretroviral therapy, and it was! initiated in these patients at their subsequent! follow-up visits. Thirty-four (20\%) patients had! already been receiving antiretroviral therapy ! before their enrollment in the study. These! observations emphasize that the prospects for! antiretroviral therapy in developing countries! have changed recently with the availability of ! cost-effective generic drugs. ${ }^{[19,20]}$ The regular! follow-up visits by these patients receiving ! antiretroviral treatment and 37 patients who! had early-stage HIV disease but did not require ! antiretroviral therapy not only indicate the ! 
antiretroviral treatment adherence but also ! the potential benefits of antiretroviral therapy ! suggest the growing awareness and motivation ! for regular HIV disease monitoring in patients in ! the developing countries.

The WHO has proposed public-health approach ! to antiretroviral therapy (ART) to enable scaling ! up of access to treatment for HIV-positive people in developing countries, recognizing ! that the Western model of specialist physician! management and advanced laboratory monitoring is not feasible in resource-poor settings. ${ }^{[21]}$ In an attempt to replicate resource-! rich CD4 count guidelines for treatment decisions and monitoring in resource-poor ! settings of developing countries like India, we studied the CD4 outcome in our study population. In 95 out of 166 HIV-infected ! individuals forming the study population, we observed a trend of increasing median CD4 counts at all subsequent follow-up visits after initiation of antiretroviral treatment as compared to the baseline median CD4 count! value, with the increase in median CD4 counts ! being more apparent in the first visit (change 1) as compared to the increase observed at subsequent follow-up visits [Figure 2]. These ! observations underscore the importance of CD4 counts in monitoring of antiretroviral treatment effectiveness even in resource-poor settings and in the presence of other problem ! background infections, thus guiding future efforts for the development of widely available, ! low-cost methods for measuring CD4 counts. ! We observed trends for increase in median CD4+ T-cell counts at subsequent follow-up visits in both the groups of $\mathrm{HIV}+\mathrm{TB}+$ and HIV+TB- patients receiving only antiretroviral ! treatment [Figure 3]. These results underscore ! in HIV-infected individuals and emphasize ! the importance of ART regimens in resolving ! CD4 lymphopenia in persistent opportunistic ! infections like TB in HIV+TB+ patients. The ! HIV $+\mathrm{TB}+$ group of 31 individuals receiving ! only treatment for tuberculosis (ATT) had! slight improvement in CD4 counts. Hence! the treatment of opportunistic infections like ! TB may not only slow the progression of HIV ! disease in patients dually infected with HIV ! and TB but may also be beneficial for patients ! facing a high risk of imminent death due to ! TB in developing countries like India. ${ }^{[22-28] !}$ These results also indicate the benefits of ! tuberculosis treatment for HIV management! in the absence of availability of antiretroviral! treatment in resource-poor settings. However, ! the group of HIV+TB+ patients $(n=10)$ ! receiving dual therapy (ART+ATT) showed! better improvement in median CD4 counts at ! follow-up visits, suggesting that a combined! better treatment modality to follow in such ! dually infected patients. Thus the initiation of ! effective antiretroviral treatment focused on ! new program-based strategies with the goal! of population-wide reduction in morbidity and ! mortality in developing countries.

!

Hence, even in the absence of standard! laboratory monitoring facilities available in ! industrialized settings, the study outcome ! presents an optimistic future outlook by ! addressing key issues of concern for the ! development of antiretroviral treatment program ! with less intense monitoring that has the ! potential to reduce morbidity and mortality from ! HIV in the high-prevalence and high-'case load' ! ART and ATT therapy regimen may be a ! clinical symptoms may also form a basis for! developing countries like India. Future research ! with larger study group and long-term follow-! up of patients may further assist the efforts ! of establishing cohorts as emphasized by the ! study outcome. This may be possible with free ! availability of antiretroviral drugs. This will also give us insight into the pathogenesis of HIV in ! infected Indian patients.

\section{REFERENCES}

1. !Forum for Collaborative HIV Research. A meeting organized by the forum for collaborative ! HIV research: Transfer of HIV diagnostic and! monitoring technologies into resource-poor! settings. Washington, DC; 22 April 2002.

2. ! Farmer P, Leandre F, Mukherjee JS, Claude M, Nevil P, Smith-Fawzi MC, et al. Community-based! approaches to HIV treatment in resource-poor ! settings. Lancet 2001;358:404-9.

3. !Weidle PJ, Malamba S, Mwebaze R, Sozi C, ! Rukundo G, Downing R, et al. Assessment of ! a pilot antiretroviral drug therapy programme in ! Uganda: patients' response, survival and drug ! resistance. Lancet 2002;360:34-40.

4. ! Adje-Toure C, Celestin B, Hanson D, Roels ! $\mathrm{TH}$, Hertogs $\mathrm{K}$, Larder $\mathrm{B}$, et al. Prevalence of ! genotypic and phenotypic HIV-1 drug-resistant strains among patients who have rebound in viral! load while receiving antiretroviral therapy in the ! UNAIDS Drug Access Initiative in Abidjan, Cote ! d'IVoire. AIDS 2003;17:S23-9.

5. ! Coetzee D, Hildebrand K, Boulle A, Maartens G, ! Louis F, Labatala V, et al. Outcomes after two years! of providing antiretroviral treatment in Khayelitsha, ! South Africa. AIDS 2004;18:887-95.

6. Hofer CB, Schechter M, Harrison LH. Effectiveness ! of antiretroviral therapy among patients who attend ! public HIV clinics in Rio de Janeiro, Brazil. J Acquir ! Immune Defic Syndr 2004;36:967-71.

7. Jaffar S, Govender T, Garrib A, Welz T, Grosskurth ! $\mathrm{H}$, Smith PG, et al. Antiretroviral treatment in ! resource-poor settings: Public health research priorities. Trop Med Int Health 2005; 10:295-9.

8. !Desvarieux M, Landman R, Liautaud B, Girard! PM. Antiretroviral therapy in resource-poor! countries: Illusions and Realities. Am J Pub Health ! 2005;95:1117-22.

9. !National AIDS Control Organization (NACO). Surveillance for HIV infection/AIDS cases in ! India. Ministry of Health and Family Welfare, ! Government of India. 1986-1999. p. 220-6.

10. Vajpayee M, Kanswal S, Seth P, Wig N, Pandey ! RM. Tuberculosis infection in HIV-infected Indian! patients. AIDS Patient Care STDs 2004;18:209-! 13.

11. Bartlett J, Fath M, DeMasi R, Quinn J, Hermes A, Rousseau F. An updated meta-analysis of triple ! combination therapy in antiretroviral-naive HIV-! infected adults. $12^{\text {th }}$ Conference on Retroviruses ! and Opportunistic Infections. Boston; February ! 22-25, 2005. Abstract 586.

12. American Thoracic Society; CDC; Infectious ! Diseases Society of America. Treatment of ! tuberculosis. MMWR Recomm Rep 2003;52:1-! 77.

13. 1997 revised guidelines for performing CD4+ T-cell determinations in persons infected with ! human immunodeficiency virus (HIV). Centers for! Disease Control and Prevention. Morbid Mortal ! Wkly Rep 1997;46:1-29.

14. Mandy F, Nicholson J, McDougal J. Revised Guidelines for performing single-platform absolute! CD4+ T-Cell determinations with CD45 gating for ! persons infected with HIV. Morbid Mortal Wkly ! Rep 2003;52:1-13.

15. Balakrishnan P, Solomon S, Kumarasamy N, ! Mayer $\mathrm{KH}$. Low-cost monitoring of HIV infected! individuals on highly active antiretroviral therapy ! (HAART) in developing countries. Indian J Med ! Res 2005;121:345-55.

16. Cecelia AJ, Balakrishnan P, Mohanakrishnan, ! Venkatakrishnan B, Solomon S, Kumarasamy N. Effective evaluation of novel low-cost CD4 monitoring ! assays. J Immunol Met 2006;316:158-62. ! 
17. Rabkin M, El-Sadr Wafaa, Katzenstein DA, ! Mukherjee J, Masur $\mathrm{H}$, Mugyenyi $\mathrm{P}$, et al. Antiretroviral treatment in resource-poor settings: ! Clinical research priorities. Lancet 2002;360:1503-! 5.

18. Kent DM, McGrath D, Ioannidis PA, Bennish ML. ! Suitable monitoring approaches to antiretroviral ! therapy in resource-poor settings: Setting the ! research agenda. Clin Infect Dis 2003;37:S13-24.

19. Garcia R, Badaro R, Netto EM, Silva M, Amorin FS, ! Ramos A, et al. Cross-sectional study to evaluate! factors associated with adherence to antiretroviral! therapy by Brazilian HIV-infected patients AIDS ! Res Hum Retroviruses 2006;22:1248-52.

20. Weiss L, French T, Waters M, Netherland J, ! Agins B, Finkelstein R. Adherence to HAART: ! Perspectives from clients in treatment support ! programs. Psychol Health Med 2006;11:155-70.

21. Gilks CF, Crowley S, Ekpini R, Grove S, Perriens! J, Souteyrand Y, et al. The WHO public-health ! approach to antiretroviral treatment against HIV in ! resource-limited settings. Lancet 2006;368:501-! 10.

22. Morris L, Martin DJ, Bredell H, Nyoka SN, Sacks ! L, Pendle S, et al. Human immuno-deficiency! virus-1 RNA levels and CD4 lymphocyte counts ! during treatment for active tuberculosis, in South ! African patients. J Infect Dis 2003;187:1967-71. 23. Hofer CB, Schechter M, Harrison LH. Effectiveness ! of antiretroviral therapy among patients who ! attend public HIV clinics in Rio de Janeiro, Brazil. J Acquir Immune Defic Syndr 2004;36:967-71.

24. O'Brien WA, Hartigan PM, Daar ES, Simberkoff ! MS, Hamilton JD. Changes in plasma HIV RNA ! levels and CD4 lymphocyte counts predict both ! response to anti retroviral therapy and therapeutic ! failure. Ann Intern Med 1997;126:939-45.

25. Grabar S, Le Moing V, Goujard C, Leport C, ! Kazatchkine MD, Costagliola D, et al. Clinical ! outcome of patients with HIV-1 infection according ! to immunologic and virologic response after 6 ! months of highly active anti retroviral therapy. Ann ! Intern Med 2000;133:401-10.

26. Mezzaroma I, Carlesimo M, Pinter E, Alario C, ! Sacco G, Muratori DS, et al. Long-term evaluation ! of T-cell subsets and T- cell functions after HAART ! in advanced stage of HIV-1 Disease. AIDS ! 1999;13:1187-93.

27. Tarwater PM, Margolick JB, Jin J, Phair JP, Detels ! $R$, Rinaldo C, et al. Increase and plateau of CD4 ! T-cell counts in the $3 \frac{1}{2}$ years after initiation of ! potent antiretroviral therapy. J Acquir Immune ! Defic Syndr 2001;27:168-75.

28. Lange CG, Lederman MM. Immune reconstitution! with antiretroviral therapies in chronic HIV-1 ! infection. J Antimicrob Chemother 2003;51:1-4.

Source of Support: Nil, Conflict of Interest: None declared. 